\title{
INTEGRAL FORMULAS FOR SUBMANIFOLDS AND THEIR APPLICATIONS
}

\author{
BANG-YEN CHEN \& KENTARO YANO
}

Various integral formulas for hypersurfaces have been established and applied to the study of closed hypersurfaces with constant mean curvature. For the literature, see [12]. Integral formulas for submanifolds of arbitrary codimensions have been obtained by Chen [4], [5], [13], Katsurada [7], [8], [9], Kôjyô [8], Nagai [9], Okumura [15], Tani [16] and Yano [5], [10], [11], [13], [15], [16]. In the present paper, we first obtain the most general integral formulas for closed submanifolds in an $m$-dimensional euclidean space, and then apply those formulas to obtain some characterizations of spherical submanifolds.

\section{Preliminaries ${ }^{1}$}

Let $M^{n}$ be an $n$-dimensional manifold with an immersion $x: M^{n} \rightarrow E^{m}$ of $M^{n}$ into a euclidean space $E^{m}$ of dimension $m$. Let $F\left(M^{n}\right)$ and $F\left(E^{m}\right)$ be respectively the bundles of orthonormal frames of $M^{n}$ and $E^{m}$. Let $B$ be the set of elements $b=\left(p, e_{1}, \cdots, e_{n}, e_{n+1}, \cdots, e_{m}\right)$ such that $\left(p, e_{1}, \cdots, e_{n}\right) \in F\left(M^{n}\right)$ and $\left(x(p), e_{1}, \cdots, e_{m}\right) \in F\left(E^{m}\right)$ whose orientation is coherent with that of $E^{m}$, by identifying $e_{i}$ with $d x\left(e_{i}\right), i=1, \cdots, n$. Define $\tilde{x}: B \rightarrow F\left(E^{m}\right)$ by $\tilde{x}(b)=$ $\left(x(p), e_{1}, \cdots, e_{m}\right)$.

Throughout this paper, we shall agree, unless otherwise stated, on the indices of the following ranges:

$$
1 \leq i, j, \cdots \leq n ; 1 \leq A, B, \cdots \leq m ; n+1 \leq r, s, \cdots \leq m .
$$

The structure equations of $E^{m}$ are given by

$$
\begin{gathered}
d x=\Sigma \omega_{A}^{\prime} e_{A}, \quad d e_{A}=\Sigma \omega_{A B}^{\prime} e_{B}, \\
d \omega_{A}^{\prime}=\Sigma \omega_{B}^{\prime} \wedge \omega_{B A}^{\prime}, \quad d \omega_{A B}^{\prime}=\Sigma \omega_{A C}^{\prime} \wedge \omega_{C B}^{\prime}, \\
\omega_{A B}^{\prime}+\omega_{B A}^{\prime}=0,
\end{gathered}
$$

where $\omega_{A}^{\prime}, \omega_{A B}^{\prime}$ are differential 1-forms on $F\left(E^{m}\right)$. Let $\omega_{A}, \omega_{A B}$ be the induced 1 -forms on $B$ from $\omega_{A}^{\prime}, \omega_{A B}^{\prime}$ by the mapping $\tilde{x}$. Then we have

\footnotetext{
Communicated November 18, 1970.

1 Manifolds, mappings, functions, ... are assumed to be differentiable and of class $C^{\infty}$, and we shall restrict ourselves only to connected submanifolds of dimension $n>1$.
} 


$$
\omega_{r}=0, \quad \omega_{i r}=\Sigma A_{r i j} \omega_{j}, \quad A_{t i j}=A_{t j i} .
$$

From (2) we can define the mean curvature vector $H$ by

$$
H=(1 / n) \Sigma A_{r i i} e_{r} .
$$

$H$ is a well-defined normal vector, and its length is called the mean curvature.

For each unit normal vector $e=\Sigma \cos \theta_{r} e_{r}$, the second fundamental form $A_{(e)}=\left(A_{i j}(e)\right)$ at $e$ is a linear transformation and is given by

$$
A_{(e)}\left(e_{i}\right)=\Sigma \cos \theta_{r} A_{r i j} e_{j}, \quad j=1, \cdots, n .
$$

If $N$ is a nonzero normal vector, then the second fundamental form at $N$ is defined as the second fundamental form at the unit direction of $N$. The principal curvatures at $e$ are defined as the eigenvalues of the second fundamental form $A_{(e)}$ at $e$. Furthermore, the $p$ th mean curvature $M_{p}(e)$ at $e$ is given by the $p$ th elementary symmetric function, i.e.,

$$
\left(\begin{array}{l}
n \\
p
\end{array}\right) M_{p}(e)=\Sigma k_{1} \cdots k_{p}
$$

where $k_{1}, \cdots, k_{n}$ are the principal curvatures at $e$, and $\left(\begin{array}{l}n \\ p\end{array}\right)=n ! /[p !(n-p) !]$. If all the principal curvatures at $e$ are the same, i.e., $k_{1}=\cdots=k_{n}$, everywhere, then $M^{n}$ is said to be umbilical with respect to $e$. If the mean curvature vector $H \neq 0$ everywhere and $M^{n}$ is umbilical with respect to $H$, then $M^{n}$ is called a pseudo-umbilical submanifold.

\section{Some integral formulas}

Let [ $\underbrace{\cdot+\cdot}$ ] denote the combined operation of exterior product and $m-1$ terms

vector product, and $($,$) the combined operation of exterior product and scalar$ product in $E^{m}$. For simple cases we have

$$
\begin{aligned}
& {\left[e_{1}, \cdots, \hat{e}_{A}, \cdots, e_{m}\right]=(-1)^{m+A} e_{A},} \\
& \begin{aligned}
&\left(v,\left[v_{1}, \cdots, v_{m-1}\right]\right)=(-1)^{m-1} \operatorname{det}\left(v, v_{1}, \cdots, v_{m-1}\right), \\
& {[\underbrace{d X, \cdots, d X}_{i}, \underbrace{d e_{n+1}, \cdots, d e_{n+1}}, e_{n+2}, \cdots, e_{m}] } \\
&=n(-1)^{m+n+1} e_{n+1} M_{i}\left(e_{n+1}\right) d V,
\end{aligned}
\end{aligned}
$$

where ${ }^{\wedge}$ denotes the omitted term, $d V=\omega_{1} \wedge \cdots \wedge \omega_{n}$ the volume element of $M^{n}$, and $X$ the position vector field of $M^{n}$ in $E^{m}$ with respect to the origin of $E^{m}$. 
The position vector field $X$ can be decomposed into two parts:

$$
X=X_{t}+X_{n},
$$

where $X_{t}$ is tangent to $x\left(M^{n}\right)$, and $X_{n}$ normal to $x\left(M^{n}\right)$. Let $e$ be a unit normal vector field over $M^{n}$, and $\bar{e}$ a unit normal vector field perpendicular to $e$ and in the direction of $X_{n}-\left(X_{n} \cdot e\right) e$, i.e.,

$$
X_{n}=\left(X_{n} \cdot e\right) e+\left(X_{n} \cdot \bar{e}\right) \bar{e}
$$

Throughout this paper, we always choose $e_{1}, \cdots, e_{n}$ in the principal directions with respect to $e$. Thus, if we denote the principal curvatures at $e$ by $k_{1}$, $\cdots, k_{n}$, then we have

$$
\omega_{i, n+1}=k_{i} \omega_{i}, \quad i=1, \cdots, n
$$

Define $n$ functions $F_{i}(e), i=1, \cdots, n$, by

$$
F_{i}(e)=\frac{(n-i) !}{n !}(X \cdot \bar{e}) \Sigma k_{j_{1}} \cdots k_{j_{i-1}} A_{j_{i} j_{i}}(\bar{e})
$$

where the summation is taken over all distinct $j_{1}, \cdots, j_{i}=1, \cdots, n$.

Suppose that the unit normal vector field $e$ is parallel in the normal bundle, i.e., by the definition, de is tangent to $x\left(M^{n}\right)$ everywhere. Then, by using (5), (6) and choosing $e_{n+1}=e$ and $e_{n+2}=\bar{e}$ everywhere, we have

$$
\begin{aligned}
d(X, & {\left.[\underbrace{d X, \cdots, d X}_{n-i}, \underbrace{d e, \cdots, d e}_{i-1}, e_{n+1}, \cdots, e_{m}]\right) } \\
= & \left.(d X, \underbrace{d X, \cdots, d X}_{n-i}, \underbrace{d e, \cdots, d e}_{i-1}, e_{n+1}, \cdots, e_{m}]\right) \\
& \left.+(-1)^{n-1}(X, \underbrace{d X, \cdots, d X}_{n-1}, \underbrace{d e, \cdots, d e}_{i}, e_{n+2}, \cdots, e_{m}]\right) \\
& +(-1)^{n-1} \sum_{s=n+2}^{m}(\underbrace{X,[\underbrace{}_{1}, \cdots, d X}_{n-i}, \underbrace{d e, \cdots, d e}_{i-1}, \\
= & (-1)^{m+i} n !\left(M_{i-1}(e)+(X \cdot e) M_{i}(e)\right) d V \\
& +(-1)^{n-1}\left(X,[\underbrace{d X, \cdots, d X}_{n-i}, d \underbrace{\left.\left.d e, \cdots, d e, e_{n+1}, d \bar{e}, e_{n+3}, \cdots, e_{m}\right]\right) .}_{i-1}\right.
\end{aligned}
$$

and 


$$
\begin{aligned}
& {[\underbrace{d X, \cdots, d X}_{n-i}, \underbrace{d e, \cdots, d e}_{i-1}, e_{n+1}, d \bar{e}, e_{n+3}, \cdots, e_{m}]} \\
& =\sum \omega_{j_{1}} \wedge \cdots \wedge \omega_{j_{n-i}} \wedge \omega_{n+1, j_{n-i+1}} \wedge \cdots \wedge \omega_{n+1, j_{n-1}} \wedge \omega_{n+2, j_{n}} \\
& \cdot\left[e_{j_{1}}, \cdots, e_{j_{n-i}}, \cdots, e_{j_{n-1}}, e_{n_{+1}}, e_{j_{n}}, e_{n+3}, \cdots, e_{m}\right] \\
& =(-1)^{i-1} \sum k_{j_{n-i+1}} \cdots k_{j_{n-1}} \omega_{j_{1}} \wedge \cdots \wedge \omega_{j_{n-i}} \wedge \cdots \wedge \omega_{j_{n-1}} \\
& \wedge \omega_{n+2, j_{n}}\left[e_{j_{1}}, \cdots, e_{j_{n-1}}, e_{n+1}, e_{j_{n}}, e_{n+3}, \cdots, e_{m}\right] \\
& =(-1)^{i+1} \sum k_{j_{1}} \cdots k_{j_{i-1}} A_{j_{i} j_{i}}(\bar{e}) \omega_{j_{1}} \wedge \cdots \wedge \omega_{j_{n}} \\
& \quad \cdot\left[e_{j_{1}}, \cdots, e_{j_{n}}, e_{n+1}, e_{n_{+3}}, \cdots, e_{m}\right] \\
& =(n-i) !(-1)^{m+n+i+1}\left(\sum k_{j_{1}} \cdots k_{j_{i-1}} A_{j_{i j} j_{i}}(\bar{e})\right) \bar{e} d V .
\end{aligned}
$$

By (8), (9) and (10) we thus get

$$
\begin{aligned}
d(X, & {\left.[\underbrace{d X, \cdots, d X}_{n-i}, \underbrace{d e, \cdots, d e}_{i-1}, e_{n+1}, \cdots, e_{m}]\right) } \\
& =(-1)^{m+i} n !\left(M_{i-1}(e)+(X \cdot e) M_{i}(e)+F_{i}(e)\right) d V, \quad i=1, \cdots, n .
\end{aligned}
$$

Hence we know that $F_{i}(e)$ are well-defined functions defined on the whole manifold $M^{n}$. By integrating both sides of the above equation and applying Stokes' theorem, we have

Proposition 2.1. Let $x: M^{n} \rightarrow E^{m}$ be an immersion of an oriented closed manifold $M^{n}$ into $E^{m}$. If $e$ is a unit normal vector field over $M^{n}$ and is parallel in the normal bundle, then

$$
\int_{M^{n}}\left(M_{i-1}(e)+(X \cdot e) M_{i}(e)\right) d V=-\int_{M^{n}} F_{i}(e) d V, \quad i=1, \cdots, n,
$$

where $M_{i}(e)$ dentoe the ith mean curvature at $e$.

In particular, we have

Theorem 2.2. Let $x: M^{n} \rightarrow E^{m}$ be an immersion of an oriented closed manifold $M^{n}$ into $E^{m}$. If $e$ is a unit normal vector field parallel in the normal bundle, and $F_{i}(e)=0$ for some $i, 1 \leq i \leq n$, then

$$
\int_{M^{n}} M_{i-1}(e) d V+\int_{M^{n}}(X \cdot e) M_{i}(e) d V=0 .
$$

Remark 2.1. If the codimension $m-n=1$, then the assumption $F_{i}(e)=0$ in Theorem 2.2 are automatically satisfied. In this case, the integral formulas (12) are called the Minkowski formulas [6].

Remark 2.2. If the codimension is greater than 1 , then in order to get some generalized Minkowski's formulas, various authors have set various assumptions. For examples: 
1. In [7], [8], [9], Katsurada, Kôjyô and Nagai assumed that the position vector field $X$ is parallel to the mean curvature vector field $H$ everywhere, and $H$ is parallel in the normal bundle. In this case, we can choose $e$ in the direction of $H$ so that $X \cdot \bar{e}=0$ by (7). Thus from (8) it follows that $F_{1}(e)=\cdots$ $=F_{n}(e)=0$ automatically.

2. In [10], [11], Yano assumed that the mean curvature vector field $H$ is parallel in the normal bundle and the second fundamental form at $X_{n}-\left(X_{n} \cdot H\right) H /(H \cdot H)$ vanishes. In this case, if we choose $e$ in the direction of $H$, then $A_{i j}(\bar{e})=0$ for all $i, j=1, \cdots, n$. Thus by (8) we know that $F_{1}(e)$ $=\cdots=F_{n}(e)=0$ automatically.

Remark 2.3. Let $e$ be a unit normal vector field. If the second mean curvature $M_{2}(e)$ is equal to an $\alpha$ th scalar curvature [3], then $e$ is called a Frenet direction [3], [5]. From the definition of $F_{i}(e)$ it follows that $F_{2}(e)=0$ if $e$ is a Frenet direction. For the integral formulas in the Frent directions see ChenYano [5]. If $e$ is parallel to $X_{n}$, then $F_{1}(e)=\cdots=F_{n}(e)=0$ automatically.

Remark 2.4. In Proposition 2.1, the condition of the parallelism of $e$ in the normal bundle can be replaced by the condition that $M^{n}$ is immersed in a hypersphere of $E^{m}$ centered at the origin of $E^{m}$.

Theorem 2.3. Let $x: M^{n} \rightarrow E^{m}$ be an immersion of an oriented closed manifold $M^{n}$ into $E^{m}$. If $e$ is a unit normal vector field and is parallel in the normal bundle, and $(X \cdot \bar{e}) A_{(\bar{e})}=0$, then we have $F_{1}(e)=\cdots=F_{n}(e)=0$ and

$$
\int_{M^{n}} M_{i-1}(e) d V+\int_{M^{n}}(X \cdot e) M_{i}(e) d V=0, \quad i=1, \cdots, n,
$$

where $A_{(\bar{e})}$ denotes the second fundamental form at $\bar{e}$.

Since $(X \cdot \bar{e}) A_{(\bar{e})}=0$, we have either $X \cdot \bar{e}=0$ or $A_{i j}(\bar{e})=0$, for all $i, j=$ $1, \cdots, n$. Thus $F_{1}(e)=\cdots=F_{n}(e)=0$ by (8), and hence we get (13) by Theorem 2.2.

Remark 2.5. If $e$ is in the direction of the mean curvature vector field $H$, then Theorem 2.3 was proved by Yano [10], [11] for $i=2$.

Let $f$ be a function on $M^{n}$. By grad $f$ or $\nabla f$ we mean $\nabla f=\Sigma f_{i} e_{i}$, where $f_{i}$ are given by $d f=\Sigma f_{i} \omega_{i}$.

Theorem 2.4. Let $x: M^{n} \rightarrow E^{m}$ be an immersion of an oriented closed manifold $M^{n}$ into $E^{m}$. If $e$ is a unit normal vector field over $M^{n}$, then we have

$$
\int_{M^{n}}\left(X \cdot \nabla M_{i}(e)\right) d V+n \int_{M^{n}}\left(M_{i}(e)+(X \cdot H) M_{i}(e)\right) d V=0,
$$

for $i=1, \cdots, n$, where $H$ denotes the mean curvature vector field.

Proof. Let

$$
\sigma=\Sigma(-)^{j-1}\left(X \cdot e_{j}\right) \omega_{1} \wedge \cdots \wedge \hat{\omega}_{j} \wedge \cdots \wedge \omega_{n} .
$$


Then

$$
d\left(M_{i}(e) \sigma\right)=\left(d M_{i}(e)\right) \wedge \sigma+n M_{i}(e)(1+(X \cdot H)) d V,
$$

which together with $d f \wedge \sigma=(X \cdot \nabla f) d V$ implies

$$
d\left(M_{i}(e) \sigma\right)=\left(X \cdot \nabla M_{i}(e)\right) d V+n M_{i}(e)(1+(X \cdot H)) d V .
$$

Hence by integrating both sides of (17) and applying Stokes' theorem, we get (14).

Remark 2.6. For the integral formulas for hypersurfaces consisting of $\nabla M_{i}(e)$, see Amur [1] and Chen [2].

\section{Some characterizations of spherical submanifolds}

The purpose of this section is to use the integral formulas in $\S 2$ to get some characterizations of spherical submanifolds. The following lemmas are wellknown.

Lemma 3.1. Let $M_{i}(e), i=1, \cdots, n$, be given by $(4)$, and let $M_{0}(e)=1$. Then

$$
M_{i}(e)^{2}-M_{i-1}(e) M_{i+1}(e) \geq 0,
$$

for $i=1, \cdots, n-1$. Moreover, if $M_{1}(e), \cdots, M_{j}(e)$ are positive, then

$$
M_{1}(e) \geq\left(M_{2}(e)\right)^{1 / 2} \geq \cdots \geq\left(M_{j}(e)\right)^{1 / j},
$$

where the equality at any stage of (18) and (19) implies that $M^{n}$ is umbilical with respect to $e$, i.e., $k_{1}=\cdots=k_{n}$

Lemma 3.2. If $M_{s-i}(e), M_{s-i-1}(e), \ldots, M_{s}(e)$ are positive, then

$$
M_{s-1}(e) / M_{s}(e) \geq M_{s-2}(e) / M_{s-1}(e) \geq \cdots \geq M_{s-i-1}(e) / M_{s-i}(e)
$$

where the equality at any stage implies that $M^{n}$ is umbilical with respect to $e$, i.e., $k_{1}=\cdots=k_{n}$.

If $x: M^{n} \rightarrow E^{m}$ is an immersion of $M^{n}$ into $E^{m}$ such that $M^{n}$ is immersed into a hypersphere of $E^{m}$ centered at $c$, then $M^{n}$ is called a spherical submanifold of $E^{m}$, or simply $M^{n}$ is spherical. The radius vector field $R$ is defined by $X-c$.

Theorem 3.3. Let $x: M^{n} \rightarrow E^{m}$ be an immersion of $M^{n}$ into $E^{m}$. Then there exists a normal vector field $e \neq 0$ over $M^{n}$ such that (1) $e$ is parallel in the normal bundle, and (2) $M^{n}$ is umbilical with respect to $e$, when and only when $M^{n}$ is spherical and $e$ is parallel to the radius vector field $R$.

Proof. Suppose that there exists a normal vector field $e \neq 0$ over $M^{n}$ such that $e$ is parallel in the normal bundle and $M^{n}$ is umbilical with respect to $e$. 
Then we can easily verify that $e$ has constant length $|e|$, and therefore we can choose the first unit normal $e_{n+1}=e /|e|$ so that

$$
\begin{aligned}
\omega_{i, n+1} & =h \omega_{i}, & i & =1, \cdots, n, \\
\omega_{n+1, r} & =0, & r & =n+1, \cdots, m .
\end{aligned}
$$

By taking the exterior derivative of (21) and applying (22), we get

$$
d h \wedge \omega_{i}=0, \quad i=1, \cdots, n,
$$

which imply that $h$ is constant. Now consider the mapping $Q: M^{n} \rightarrow E^{m}$ defined by $Q(p)=x(p)+e_{n+1} / h$. Then use of (21) and (22) yields $d Q(p)=0$, which shows that

$$
x(p)+e_{n+1} / h=c=\text { constant } .
$$

Hence $M^{n}$ is immersed into a hypersphere of $E^{m}$ centered at $c$, and $e$ is parallel to the radius vector field $X-c$.

Corollary 3.1. Let $x: M^{n} \rightarrow E^{n+2}$ be an immersion of $M^{n}$ into $E^{n+2}$. Then there exists a unit normal vector field $e$ such that $M^{n}$ is umbilical with respect to $e$ and the first mean curvature $M_{1}(e)$ at $e$ is constant when and only when $M^{n}$ is spherical and $e$ is parallel to the radius vector field $R$.

Proof. Suppose that $e$ is a unit normal vector field such that $M^{n}$ is umbilical with respect to $e$, and the first mean curvature $M_{1}(e)$ at $e$ is constant. Then by choosing $e_{n+1}=e$ we have

$$
\omega_{i, n+1}=M_{1}(e) \omega_{i}, \quad i=1, \ldots, n .
$$

By taking the exterior derivative of the above equations we get

$$
d M_{1}(e) \wedge \omega_{i}=\omega_{i, n+2} \wedge \omega_{n+2, n+1}=0 .
$$

Thus

$$
\omega_{i, n+2}=0, \quad i=1, \cdots, n,
$$

on the set $U=\left\{p \in M^{n}: \omega_{n+1, n+2} \neq 0\right.$ at $\left.p\right\}$. On the open set $U$, by taking exterior derivative of the above equations, we have

$$
\omega_{i} \wedge \omega_{n+1, n+2}=0, \quad i=1, \cdots, n,
$$

which imply that $\omega_{n+1, n+2}=0$ on $U$. Therefore $U$ is an empty set. This shows that $e$ is parallel in the normal bundle. Hence, by using Theorem 3.3, we know that $M^{n}$ is spherical and $e$ is parallel to the radius vector field $R$.

Throughout the remainder of this paper, we always assume that $x: M^{n} \rightarrow E^{m}$ is an immersion of an oriented closed manifold $M^{n}$ into $E^{m}$. 
Theorem 3.4. If there exist a unit normal vector field $e$ parallel in the normal bundle and an integer $i, 2 \leq i \leq n$, such that

(i) $M_{i}(e)>0$,

(ii) $X \cdot e \leq-M_{i-1}(e) / M_{i}(e)$, (or $X \cdot e \geq-M_{i-1}(e) / M_{i}(e)$ ),

(lii) $F_{i}(e)=F_{i-1}(e)=0$,

then $M^{n}$ is spherical and the radius vector field $R$ is parallel to $e$.

Proof. Suppose that there exist a unit normal vector field $e$ parallel in the normal bundle and an integer $i, 1<i \leq n$, such that (i), (ii) and (iii) hold. Then by (ii) and Theorem 2.2 we get

$$
\begin{gathered}
X \cdot e=-M_{i-1}(e) / M_{i}(e), \\
\int_{M^{n}}\left(M_{i-2}(e)+(X \cdot e) M_{i-1}(e)\right) d V=0,
\end{gathered}
$$

and threfore

$$
\int_{M^{n}}\left(1 / M_{i}(e)\right)\left(M_{i-1}(e)^{2}-M_{i-2}(e) M_{i}(e)\right) d V=0 \text {. }
$$

Thus by Lemma 3.1 we have $M_{i-1}(e)^{2}-M_{i-2}(e) M_{i}(e)=0$, and $M^{n}$ is umbilical with respect to $e$. Hence Theorem 3.4 follows immediately from Theorem 3.3.

Theorem 3.5. If there exist a unit normal vector field $e$ parallel in the normal bundle and an integer $i, 1<i<n$, such that

(i) $M_{i+1}(e)>0$,

(ii) $X \cdot e \geq-M_{i-1}(e) / M_{i}(e)$,

(iii) $F_{i+1}(e)=0$,

then $M^{n}$ is spherial and $e$ is parallel to the radius vector field.

Proof. By (ii) and Lemma 3.2 we have

$$
X \cdot e \geq-M_{i-1}(e) / M_{i}(e) \geq-M_{i}(e) / M_{i+1}(e),
$$

which, together with

$$
\int_{M^{n}}\left(M_{i}(e)+(X \cdot e) M_{i+1}(e)\right) d V=0
$$

by Theorem 2.2, implies

$$
X \cdot e \geq-M_{i-1}(e) / M_{i}(e) \geq-M_{i}(e) / M_{i+1}(e)=X \cdot e .
$$

Therefore $M_{i}(e)^{2}=M_{i-1}(e) M_{i+1}(e)$, and hence by Theorem 3.3, $M^{n}$ is spherical and $e$ is parallel to the radius vector field. 
Theorem 3.6. If there exist a unit normal vector field e parallel in the normal bundle and two integers $s$ and $i, 1 \leq i<s \leq n$, such that

(i) $M_{s}(e), \cdots, M_{i}(e)>0$,

(ii) $M_{s}(e)=\sum_{j=i}^{s-1} c_{j} M_{j}(e)$, for some constants $c_{j}, i \leq j \leq s-1$,

(iii) $F_{j}(e)=0, j=i, \cdots, s-1$,

then $M^{n}$ is spherical and $e$ is parallel to the radius vector field.

Proof. By Lemma 3.2 we have

$$
\frac{M_{j}(e)}{M_{s}(e)}-\frac{M_{j-1}(e)}{M_{s-1}(e)}=\left(\frac{M_{j}(e)}{M_{s-1}(e)}\right)\left(\frac{M_{s-1}(e)}{M_{s}(e)}-\frac{M_{j-1}(e)}{M_{j}(e)}\right) \geq 0,
$$

for $i \leq j \leq s-1$, so that

$$
1=\sum c_{j} M_{j}(e) / M_{s}(e) \geq \sum c_{j} M_{j-1}(e) / M_{s-1}(e)
$$

or

$$
M_{s-1}(e)-\sum c_{j} M_{j-1}(e) \geq 0,
$$

where equality holds only if $M^{n}$ is umbilical with respect to $e$. Thus by using (ii) and Theorem 2.2 we have

$$
\begin{aligned}
\int_{M^{n}}\left(M_{s-1}(e)-\sum_{j=i}^{s-1} c_{j} M_{j-1}(e)\right) d V \\
=-\int_{M^{n}}(X \cdot e)\left(M_{s}(e)-\sum_{j=i}^{s-1} c_{j} M_{j}(e)\right) d V=0,
\end{aligned}
$$

and therefore $M_{s-1}=\sum c_{j} M_{j-1}(e)$, which implies that $M^{n}$ is umbilical with respect to $e$. Hence by Theorem 3.3, $M^{n}$ is spherical and $e$ is parallel to the radius vector field.

Similarly, by using (23), we have

Theorem 3.7. If there exist a unit vector field $e$ parallel in the normal bundle and two integers $s$ and $i, 0 \leq i<s<n$, such that

(i) $M_{s+1}(e), \cdots, M_{i+1}(e)>0$,

(i!) $M_{s}(e)=\sum_{j=i}^{s-1} c_{j} M_{j}(e)$ for some constants $c_{j}, i \leq j \leq s-1$,

(iii) $X \cdot e>0$ or $X \cdot e<0$,

(iv) $F_{s-1}(e)=\cdots=F_{i}(e)=0$,

then $M^{n}$ is spherical and $e$ is parallel to the radius vector field.

Theorem 3.8. If there exist a unit normal vector field $e$ parallel in the normal bundle and an integer $i, 1 \leq i \leq n$, such that 
(i) $M_{i}(e)>0$,

(ii) $M_{i}(e)=c M_{i-1}(e)$, for a constant $c$,

(iii) $F_{i-1}(e)=F_{i}(e)=0$,

then $M^{n}$ is spherical and $e$ is parallel to the radius vector field.

Proof. Since $M_{i}(e)>0, c$ cannot be zero and $M_{i-1}(e)$ must be of fixed sign. By (ii) and Lemma 3.1, we have

$$
M_{i-1}(e)\left(M_{i-1}(e)-c M_{i-2}(e)\right)=M_{i-1}(e)^{2}-M_{i}(e) M_{i-2}(e) \geq 0,
$$

from which, together with (ii) again and Theorem 2.2, it follows

$$
\int_{M^{n}}\left(M_{i-1}(e)-c M_{i-2}(e)\right) d V=\int_{M^{n}}\left(c M_{i-1}(e)-M_{i}(e)\right)(X \cdot e) d V=0,
$$

so that $M_{s-1}(e)=c M_{s-2}(e)$. Thus by Theorem 3.6, $M^{n}$ is umbilical with respect to $e$, and hence $M^{n}$ is spherical and $e$ is parallel to the radius vector field.

Theorem 3.9. If there exists a unit normal vector field $e$ parallel in the normal bundle such that

(i) $M_{n}>0$,

(ii) the sum of the principal radii of curvature is constant at e, i.e., $\sum_{i=1}^{n}\left(1 / k_{i}\right)=$ constant,

(iii) $F_{n}(e)=F_{n-1}(e)=0$,

then $M^{n}$ is spherical and $e$ is parallel to the radius vector field.

Proof. Since

$$
\sum_{i=1}^{n}\left(1 / k_{i}\right)=n M_{n-1}(e) / M_{n}(e)=\text { constant },
$$

by Theorem 3.8 we know that $M^{n}$ is spherical and $e$ is parallel to the radius vector field.

Remark 3.1. If we replace $e$ by a unit vector field parallel to the mean curvature vector field $H$, then the conclusion in Theorems 3.3 to 3.9 and Corollary 3.1 should be read as " $M^{n}$ is a minimal submanifold of a hypersphere of $E^{m}$ ", since the only closed submanifolds of a euclidean space such that the position vector field parallel to the mean curvature vector field $H$ everywhere are the minimal submanifolds of a hypersphere.

Remark 3.2. If $M^{n}$ is spherical and $e$ is a unit vector field parallel to the radius vector field $R$, then we have (i) $e$ is parallel in the normal bundle, (ii) $M_{i}(e)=$ constant, for all $i=1, \cdots, n$, (iii) $R \cdot e= \pm M_{i}(e) / M_{i-1}(e)=$ constant, for all $i=1, \cdots, n$, and (iv) $F_{i}(e)=\cdots=F_{n}(e)=0$.

Remark 3.3. If the ambient space is replaced by an $m$-dimensional Riemannian space of constant sectional curvature and the position vector field $X$ replaced by a concurrent vector field over $M^{n}$, then we can get the same 
results for all theorems in $\S \S 2$ and 3 except the statement " $M^{n}$ is spherical and $e$ is parallel to the radius vector field" should be replaced by the existence of a concurrent normal vector field (see Yano-Chen [14]).

Remark 3.4. Let $N$ be a vector field in $E^{m}$ over $M^{n}$. If there exist a function $f$ and a 1 -form $\lambda$ such that $d(x+f N)=\lambda N$, then the vector field $N$ is called a torse-forming, because if we develop the vector field $N$ along a curve in the manifold $M^{n}$, we obtain a field of vectors along the curve whose prolongations are tangent to another curve. From Theorem 3.3 it follows that a normal vector field $N$ in $E^{m}$ over $M^{n}$ is a concurrent vector field if it is a torseforming.

\section{References}

[1] K. Amur, Vector forms and integral formulas for hypersurfaces in Euclidean space, J. Differential Geometry 3 (1969) 111-123.

[2] B. Y. Chen, Some integral formulas for the hypersurfaces in euclidean space, Nagoya Math. J. 43 (1971).

[ 3 ] - - On the scalar curvature of immersed manifolds, Math. J. Okayama Univ. 15 (1971).

[4] - Pseudo-umbilical surfaces in euclidean spaces, Kōdai Math. Sem. Rep., to appear.

[ 5 ] B. Y. Chen \& K. Yano, Pseudo umbilical submanifolds in a Riemannian manifold of constant curvature, to appear.

[6] C. C. Hsiung, Some integral formulas for closed hypersurfaces, Math. Scand. 2 (1954) 286-294.

[ 7 ] Y. Katsurada, Closed submanifolds with constant $v$-th mean curvature related with a vector field in a Riemannian manifold, J. Fac. Sci. Hokkaido Univ. (I) 20 (1969) 171-181.

[ 8 ] Y. Katsurada \& H. Kôjyô, Some integral formulas for closed submanifolds in a Riemann space, J. Fac. Sci. Hokkaido Univ. (I) 20 (1968) 90-100.

[9] Y. Katsurada \& T. Nagai, On some properties of a submanifold with constant mean curvature in a Riemann space, J. Fac. Sci. Hokkaido Univ. (I) 20 (1968) 79-89.

[10] K. Yano. Integral formulas for submanifolds and their applications, Canad. J. Math. 22 (1970) 376-388.

[11] - Submanifolds with parallel mean curvature vector of a euclidean space or a sphere, Kc̄dai Math. Sem. Rep., to appear.

[12] - Integral formulas in Riemannian geometry, Marcel Dekker, New York, 1970.

[13] K. Yano \& B. Y. Chen, Minimal submanifolds of a higher dimensional sphere, Tensor, to appear.

[14] - On the concurrent vector fields of immersed manifolds, Kōdai Math. Sem. Rep., to appear.

[15] K. Yano \& M. Okumura, Integral formulas for submanifolds of codimension 2 and their applications, Kōdai Math. Sem. Rep. 21 (1969) 463-471.

[16] K. Yano \& M. Tani, Submanifolds of codimension 2 in a euclidean space, to appear.

Michigan State University TOKYo Institute of TeChNOLOgY 
